\title{
Genetic and clinical analysis in a Chinese parkinsonism-predominant spinocerebellar ataxia type 2 family
}

\author{
Hao Sun ${ }^{1}$, Wataru Satake ${ }^{2}$, Changjun Zhang ${ }^{3}$, Yoshitaka Nagai ${ }^{2}$, Youyong Tian ${ }^{4}$, Shouzhi $\mathrm{Fu}^{4}$, Jiankun $\mathrm{Yu}^{1}$, \\ Yaping Qian ${ }^{1}$, Yuan Qian ${ }^{1}$, Jiayou $\mathrm{Chu}^{1}$ and Tatsushi Toda ${ }^{2}$
}

Parkinson's disease is a degenerative central nervous system disorder that often impairs motor skills, speech and other functions. We discovered a large Chinese family showing primarily parkinsonism symptoms with autosomal dominant inheritance. Six affected individuals in the family showed typical parkinsonism symptoms, including pill-rolling tremor. Two other affected individuals showed cerebellar ataxia symptoms. A whole-genome scan using the $50 \mathrm{~K}$ single nucleotide polymorphism array with three different linkage methods detected two positive regions on chromosome 12q24.1 and 5q13.3. The ATXN2 gene, responsible for spinocerebellar ataxia type 2 (SCA2) was located precisely in the center of the positive region on chromosome 12. Further analysis of SCA2 revealed heterozygous pathological CAG expansions in the family. The affected individuals' symptoms were typical of parkinsonism, but complex. Inverse correlation between CAG repeat size and age of onset is not obvious in this pedigree. This parkinsonism-predominant SCA2 family shared the same disease gene locus with other 'standard' SCA2 families, but it is possible that variations in one or more modifier genes might account for the parkinsonism-predominant SCA2 predisposition observed in this pedigree.

Journal of Human Genetics (2011) 56, 330-334; doi:10.1038/jhg.2011.14; published online 10 February 2011

Keywords: 5q13; genome-wide scan; linkage study; single nucleotide polymorphism chip

\section{INTRODUCTION}

Parkinson's disease (PD), a degenerative central nervous system disorder, often impairs motor skills, speech and other functions. ${ }^{1}$ It is found worldwide, with incidence rates varying from country to country. The prevalence of PD increases with age. In Europe, PD affects about $1-2 \%$ of individuals over 60 years of age. ${ }^{2}$ Although there is no cure for $\mathrm{PD}$, further understanding of its genetic risks can improve neuroprotective or preventive approaches. Causative genes for Mendelian-inherited parkinsonism have been identified. Point mutations and multiplications in the SNCA gene have been found in some families with autosomal dominant inheritance. ${ }^{3,4}$ To date, mutations in the LRRK2 gene are the most common cause of Mendelian PD. In studies across several populations, 5-15\% of autosomal dominant PD families carried mutations in LRRK2 (see refs 5,6). Mutations in three genes, PARK2 (encoding parkin), PINK1 (PARK6) and DJ-1 (PARK7), have been identified in autosomal recessive $\mathrm{PD}$, which is characterized by an early age at onset and therefore referred to as autosomal recessive juvenile parkinsonism. ${ }^{7-9}$ The expanded ATXN2 gene, which causes spinocerebellar ataxia type 2 (SCA2), was found in some families with only or mainly typical parkinsonism. ${ }^{10,11}$ Although some parkinsonism clinical signs such as dystonia and tremor have been described in SCA2, dopamine-responsive parkinsonism has been infrequently described in SCA2 (see ref. 12). The sign of dopamine-responsive just has been seen in some Chinese families ${ }^{13,14}$ and some white families. ${ }^{15,16}$

We described here a large family from Hubei, China, that showed primarily autosomal dominant inheritance of parkinsonism symptoms acrossing four generations. Affected family members exhibited typical clinical features of $\mathrm{PD}$, such as pill-rolling tremors and levodopa responsiveness. However, some family members showed cerebellar symptoms. The patients who showed the atypical phenotypes opposed to the typical cerebellar ataxia maybe have more complex genetic causes than normal SCA2 patients. So, we performed a whole-genome linkage study to identify possible genetic causes in

\footnotetext{
${ }^{1}$ The Department of Medical Genetics, Institute of Medical Biology, Chinese Academy of Medical Sciences and Peking Union Medical College, Yunnan, China; ${ }^{2}$ Division of Neurology/Molecular Brain Science, Kobe University Graduate School of Medicine, Kobe, Japan; ${ }^{3}$ Institute of Eugenics and Genetics, Hubei, China and ${ }^{4}$ Emergency Department, the Peoples Hospital of Shiyan, Hubei, China

Correspondence: Professor T Toda, Division of Neurology/Molecular Brain Science, Kobe University Graduate School of Medicine, 7-5-1 Kusunoki-chou, Chuo-ku, Kobe 650-0017, Japan.

E-mail: toda@med.kobe-u.ac.jp

or Professor J Chu, The Department of Medical Genetics, Institute of Medical Biology, Chinese Academy of Medical Sciences and Peking Union Medical College, 379 Jiaolin Road, Kunming, Yunnan 650118, China.

E-mail: chujy@imbcams.com.cn
}

Received 29 November 2010; revised 10 January 2011; accepted 13 January 2011; published online 10 February 2011 
this family. At same time, the molecular and clinical features of this family were analyzed.

\section{MATERIALS AND METHODS}

\section{Clinical information}

The proband (IV-3) was initially diagnosed as PD in 2001, and therefore the family was classified as a PD pedigree. In 2007, we performed neurological examinations for eight patients in the family, and we examined four patients using magnetic resonance imaging (MRI). Blood samples were obtained from patients and unaffected relatives with informed consent. Approval for the study was obtained from the Ethical Committees of participating institutions.

Whole-genome linkage analysis

Genomic DNA was isolated from blood using QIAamp DNA Blood Mini Kits (Qiagen, Shanghai, China). Single nucleotide polymorphism genotyping was performed for 27 individuals from the family (Figure 1) using the Human Mapping 50K Xba 240 SNP array (Affymetrix, Santa Clara, CA, USA). Signal intensity data were analyzed using GeneChip DNA analysis software GDAS v.3.0.2.8 (Affymetrix). The genotype data were converted to linkage format using ALOHOMORA software ${ }^{17}$ and subjected to quality control routines, including gender check and graphical representation of relationship errors. ${ }^{18}$ Mendelian errors were detected with PedCheck, ${ }^{19}$ and non-informative markers were deleted before further analysis. Genome-wide non-parametric multipoint linkage, single parametric and single non-parametric linkage analysis were performed using GeneSpring GT software (Agilent, Santa Clara, CA, USA).

\section{Trinucleotide repeat analysis}

We screened for mutations in the ATXN2 gene using PCR amplification with previously published SCA-2A and SCA-2B oligonucleotide primers. ${ }^{20}$ PCR products were sized precisely using capillary electrophoresis with an ABI 3730xL DNA analyzer (Applied Biosystems, Foster city, CA, USA) and compared with known samples using GeneMapper V3.5 (Applied Biosystems, Foster city, CA, USA). Some samples were isolated from agarose gels and used as DNA templates for sequencing with the Big-dye terminator kit (Applied Biosystems) on the ABI 3730xL analyzer.

\section{RESULTS}

\section{Clinical information}

The family (Figure 1) consisted of 39 members in four generations, with 16 affected members. All family members reside in Hubei Province, China. The inheritance pattern is autosomal dominant. We collected peripheral blood from 27 family members, including 10 affected members. Clinical data were shown in Table 1 for 8 of 10 patients collected in 2007. Detailed clinical data were unavailable for

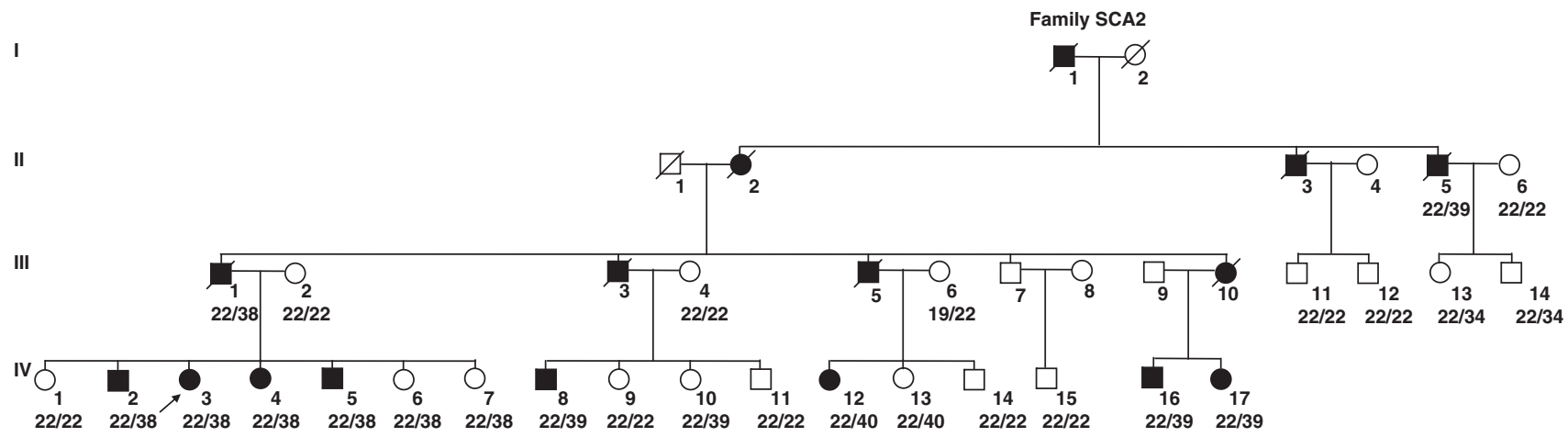

Figure 1 Pedigree of a Chinese family ascertained with parkinsonism-predominant spinocerebellar ataxia type 2 (SCA2). Squares indicate males; circles, females. A slash through the symbol indicates deceased and an arrow points to the proband. The pedigree contains 16 known affected individuals; eight patients are living. SCA2 CAG repeat allele sizes are listed below the pedigree symbols of the 27 individuals who have been genotyped.

Table 1 Clinical and genetic features of the SCA2 family

\begin{tabular}{|c|c|c|c|c|c|c|c|c|}
\hline No. & $I V-2$ & $I V-3$ & $I V-4$ & $I V-5$ & IV-8 & IV -12 & IV-16 & IV-17 \\
\hline Age at onset & 33 & 36 & 37 & 22 & 20 & 37 & 39 & 35 \\
\hline Age at examination & 46 & 44 & 42 & 39 & 50 & 45 & 51 & 38 \\
\hline Resting tremor & 2 & 1 & 0 & 2 & 0 & 0 & $2^{a}$ & 0 \\
\hline Bradykinesia & 3 & 2 & 0 & 3 & 0 & 1 & 2 & 2 \\
\hline Rigidity & 3 & 2 & 0 & 2 & 0 & 1 & 2 & 1 \\
\hline Postural instability & 2 & 1 & 0 & 2 & 0 & 2 & 2 & 1 \\
\hline Masked face & 3 & 2 & 0 & 3 & 1 & 2 & 2 & 2 \\
\hline Levodopa response & + & + & - & NT & NT & + & + & NT \\
\hline Gait ataxia & 0 & 0 & 2 & 1 & 0 & 0 & 0 & 0 \\
\hline Limb ataxia & 1 & 1 & 2 & 1 & 0 & 0 & 0 & 0 \\
\hline Slow saccade & 2 & 1 & 1 & 2 & $3^{b}$ & 1 & 1 & 0 \\
\hline Vertical gaze palsy & 2 & 0 & 0 & 2 & $3^{b}$ & 0 & 0 & 0 \\
\hline Hyporeflexia & 3 & 3 & 1 & 0 & 1 & 0 & 0 & 0 \\
\hline Cerebellar atrophy on MRI & 2 & 1 & 3 & 1 & NT & NT & NT & NT \\
\hline CAG repeats & $22 / 38$ & $22 / 38$ & $22 / 38$ & $22 / 38$ & $22 / 39$ & $22 / 40$ & $22 / 39$ & $22 / 39$ \\
\hline
\end{tabular}

Abbreviations: MRI, magnetic resonance imaging; SCA2, spinocerebellar ataxia type 2.

apill-rolling tremor.

bdifficulty initiating pursuit movements.

0 indicates that the individual was tested and the symptom was absent. 1, mild; 2 , moderate; and 3, marked. NT indicates that the individual could not be tested. A (+) indicates that the finding was present; a (-) indicates absent. 

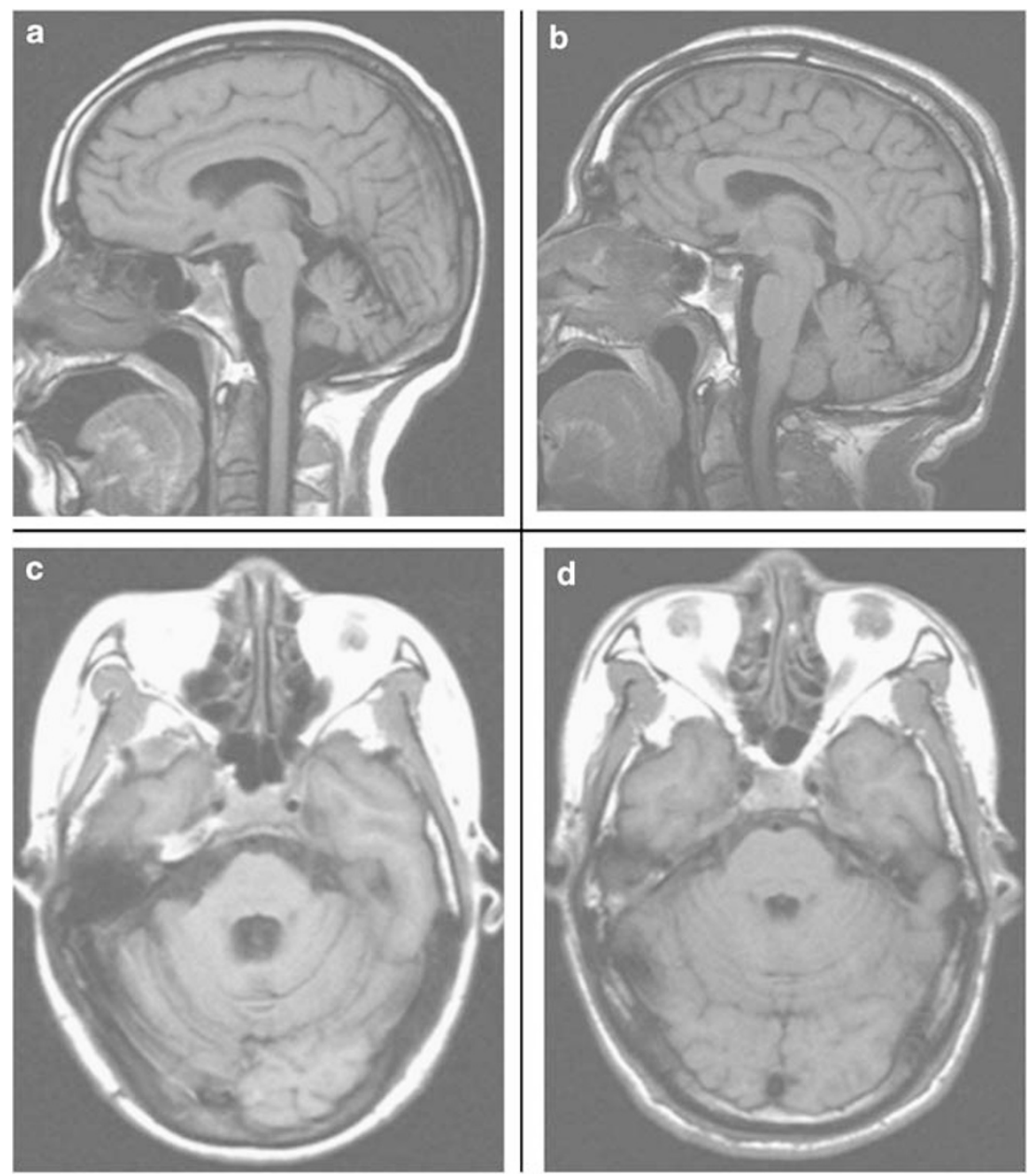

Figure 2 T1-weighted magnetic resonance imaging (MRI) of IV-4 (a, c) and IV-5 (b, d). Patient IV-4 showed marked cerebellar atrophy, and patient IV-5 showed no cerebellar atrophy.

the other two deceased patients (II-5 and III-1). Family members of the two patients provided ambiguous clinical data, and their preliminary diagnosis indicated that the two patients had parkinsonism symptoms. Blood samples, collected in 2001, were provided by their neurologist. Age of symptomatic disease onset varied from 20 to 39 years, with an average age at onset of 32.4 years.

Most patients showed typical parkinsonism symptoms, such as resting tremor, bradykinesia, rigidity and postural instability (IV-2, IV-3, IV-5, IV-12, IV-16 and IV-17). Patient IV-16 had pill-rolling tremors. However, two patients (IV-4 and IV-8) showed cerebellar symptoms such as limb ataxia and slow saccade, but no typical parkinsonism symptoms.

Five of eight patients were treated with levodopa. Only one (Patient IV-4) of them showed no response. This patient also lacked typical parkinsonism symptoms. Patients IV-2, IV-3, IV-4 and IV-5 were examined using MRI analysis. MRI images from Patients IV-4 and IV-5 were shown in Figure 2. Marked cerebellar atrophy was found in Patient IV-4, and no cerebellar atrophy was found in Patient IV-5.

\section{Whole-genome linkage analysis}

Genome-wide analysis revealed two positive regions for linkage, on chromosomes 12 and 5. On chromosome 12, the highest single-point parametric log of odds (LOD) score (2.59) was detected at rs2695281 (100.5 Mb, NCBI Build 36). The highest multi-point non-parametric
LOD score on chromosome 12 was 3.5 , with a multi-point nonparametric LOD score $>3$ at $94.9-115.6 \mathrm{Mb}$ (NCBI Build 36). On chromosome 5, the highest single-point parametric LOD score (2.73) was detected at rs10491487 (80.4 Mb, NCBI Build 36). The highest multi-point non-parametric LOD score on chromosome 5 was 3.5 with a multi-point non-parametric LOD score $>3$ at $79.8-81.2 \mathrm{Mb}$ (NCBI Build 36). Multi-point non-parametric linkage results were compatible with the single-point parametric linkage results on both chromosomes 5 and 12. Single-point non-parametric linkage analysis detected no positive result. Most of single-point non-parametric LOD scores were smaller than 2 , and the highest was 2.1, on chromosome 21 (Figure 3). Gender and relationships in the single nucleotide polymorphism array data were accurate, and PedCheck detected no Mendelian errors.

The ATXN2 gene, responsible for SCA2, was located at $110.3 \mathrm{Mb}$ (NCBI Build 36) on chromosome 12, within the linkage-positive region of $94.9-115.6 \mathrm{Mb}$.

\section{Trinucleotide repeat analysis}

We performed SCA2 molecular analysis in all 27 family members. Three types of heterozygous pathological CAG expansion $(38,39$ and 40 repeats) and two types of normal repeats (19 and 22) were identified. A borderline 34 CAG repeat was found in the two family members. Genotyopes with repeat numbers for individual family 


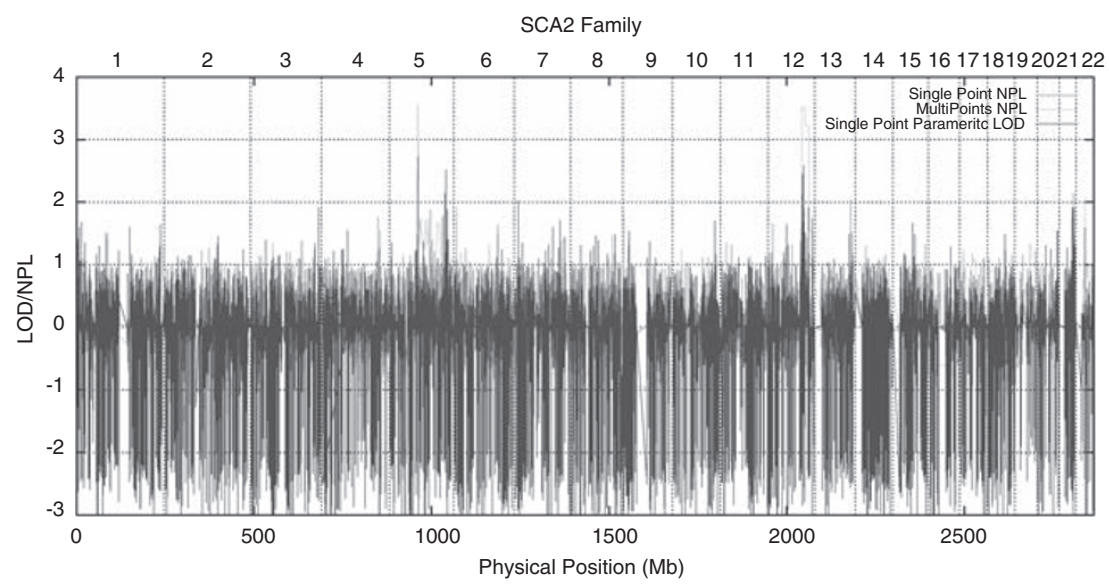

Figure 3 Three types of log of odds (LOD) scores were calculated from the genome-wide scan. The LOD score plot was created with GNUPLOT 4.0 (http:// www.gnuplot.info) using the LOD plot-drawing Perl script included in the ALOHOMORA package. On chromosome 12, the highest MultiPoint non-parametric LOD score was 3.5 and the highest SinglePoint parametric LOD score was 2.59. On chromosome 5, the highest MultiPoint non-parametric LOD score was 3.5 and the highest SinglePoint parametric LOD score was 2.73. The MultiPoint non-parametric linkage results were compatible with the SinglePoint parametric linkage results on chromosomes 5 and 12. LOD scores $<-3$ are not shown.

members were shown in Figure 1. Sequence analysis of expanded alleles from IV-5, IV-8, IV-12 and III-14 revealed interruptions of CAG base pair repeats with CAA. The 38-repeat expansion in IV-5 contained 29 CAGs, followed by one CAA and eight CAGs. The 39-repeat expansion IV-8 contained 30 CAGs, one CAA and eight CAGs. The 40-repeat expansion in IV-12 contained 31 CAGs, one CAA and eight CAGs. The normal 34-repeat CAG expansion in III-14 contained 25 CAGs, one CAA and eight CAGs.

All affected individuals in the branch of III-1 carried 38 trinucleotide repeats. IV-6 and IV-7 also carried the 38 trinucleotide repeats, but as of 2007 no parkinsonism or ataxia had been observed in these individuals. The 39 trinucleotide repeats occurred in the branches of II-5, III-3 and III-10. Similar to IV-6 and IV-7, individual IV-10 carried the 39 trinucleotide repeats, but had shown no parkinsonism or ataxia symptoms in 2007. In the branch of II-5, the 39 repeats were shortened to 34 in transmission. III-13 and III-14, who carried 34 trinucleotide repeats, showed no symptoms.

\section{DISCUSSION}

We described a levodopa-responsive parkinsonism Chinese family with SCA2 trinucleotide expansions. The symptoms observed in this family were primarily parkinsonism, but complex. Some affected family members showed typical clinical manifestations of parkinsonism. Four of five patients responded to levodopa treatment. One affected individual (IV-4) lacking parkinsonism symptoms was unresponsive to levodopa; MRI analysis of this patient showed marked cerebellar atrophy. Patient IV-8 showed just mild masked face, but his cerebellar symptoms were severe. In other patients (IV-12, IV-16 and IV-17), cerebellar symptoms were minor or absent. Overall, the clinical signs in this family appear most similar to parkinsonism.

Molecular analysis of SCA2 expansion in the family revealed three types of expanded CAG repeats. An inverse correlation has been established between age of SCA2 onset and CAG repeat length, with repeat length accounting for $54-80 \%$ of variance. ${ }^{21,22}$ However, such inverse correlation was not observed in our pedigree. In the branches of III-1 and III-2, the age of onset in affected individuals who carried the 38 CAG repeats ranged from 22 to 37 . Two other individuals with 38 CAG repeats showed no clinical signs at the time of examination (IV-6, age 37 years in 2007; and IV-7, age 35 years in 2007). The age of onset in the three affected individuals who carried 39 CAG repeats
(II-5, IV-16 and IV-17) ranged from 20 to 39 years. Another carrier of 39 CAG repeats (IV-10) showed no clinical signs in 2007, at age 43 years. One of the two individuals who carried 40 CAG repeats had an age at onset of 37 years (IV-12). The other (IV-13) had no clinical signs in 2007, at age 43 years. These observations showed that repeat length alone cannot account for age of onset in this family. Conversely, it is not possible to predict when, or if, the unaffected carriers of expanded CAG repeats will eventually show clinical signs.

Some researches suggested that CAA interruption can lead to phenotypical variation. ${ }^{23,24}$ The results of Sobczak et al. ${ }^{25}$ showed that the CAA interruptions are major determinants of the CAG repeat folding in the SCA2 transcripts. The SCA 2 transcripts interrupted by the CAA should generate shorter branched hairpins and the uninterrupted repeats transcripts form single slippery hairpins. The patients who carried SCA2 expansions with and without interruptions show two different phenotypes. ${ }^{23,24}$ It may be caused by the different CAG repeat folding that would interact differently with doublestranded RNA binding proteins and interfere with mRNA transcription or translation. ${ }^{24}$ That structural organization of CAG expansions with interruption associate with phenotypic variation has been also reported in other neurodegenerative disorders such as SCA1 (see ref. 26). In our family, all patients carried the CAG expansions with one CAA interruption, but showed two different phenotypes. The patients IV-4 and IV-8 showed more ataxia symptoms than the other patients. Especially, patient IV-4 had no response to the levodopa treatment and had marked cerebellar atrophy on MRI. The symptoms of IV-4 made him look more like the typical SCA2. Therefore, the phenotypic variation in our family may be caused by other unknown reason such as co-effect of SCA2 gene and some modifier gene, rather than the different CAA interruption.

The SCA2 CAG repeat is highly unstable through intergenerational transmission, with a tendency to expand. One study reported that there are 27 families' SCA2 CAG repeats changed in length among 32 SCA2 families, with a mean increase of 2.2 repeat units. ${ }^{27}$ In this family, we observed eight transmissions of an expanded SCA2 CAG repeat with no increase in repeat length. Six transmissions yielded no change, whereas two transmissions yielded contractions. As blood samples from several older patients were not collected, we cannot know exactly which SCA2 CAG repeat increased through intergenerational transmission. The explanation for the relative instability in the 
family may include genetic or epigenetic factors. A previous study defined the range of the normal SCA2 allele size as 17-31 CAG repeats, whereas full pathogenic mutations had $36-64$ repeats. ${ }^{28}$ Two unaffected family members who carried the contracted CAG repeat had borderline mutations of 34 CAG repeats (III-13, 45 years of age in 2007; and III-14, 38 years of age in 2007), although 34 CAG repeats were found in some SCA2 patients. ${ }^{29}$

Our linkage analysis revealed two positive regions, one at chromosome $12 \mathrm{q} 24.1$ and one at chromosome $5 \mathrm{q} 13.3$. On chromosome 12, the mutated ATXN2 gene, which causes SCA2, is located in the middle of the linkage-positive region. This demonstrates the accuracy of the detection methods and shows that the positive regions are reliable. As the disease gene of SCA2 was located to chromosome 12q23-24.1 (see ref. 30), most of the reports about parkinsonism-predominant SCA2 was based on case cohorts. The linkage study in our family strengthened that the parkinsonism-predominant SCA2 carried the same pathogenic gene as the typical SCA2 from the whole genome perspective. We assume that this parkinsonism-predominant SCA2 family shares a disease locus with other standard SCA2 families, but it is possible that one or more modifier genes interact with ATXN2 to produce clinical signs more similar to parkinsonism. It is difficult to predict whether the other positive region at $5 \mathrm{q} 13.3$ is a real positive region or an artifact. If it is not an artifact, perhaps there would be a modifier gene harbored in the region. To prove it, more detailed gene mutation analyses in the region or other linkage analyses for additional parkinsonism-predominant SCA2 families need to be performed.

The prevalence of SCA2 among patients with familial parkinsonism ranged from 1.5 to $10 \%$ (ref. 12). It is seen occasionally in German ${ }^{28}$ and Japanese ${ }^{10}$ populations. After Gwinn-Hardy et al. described a Chinese American family with only or mainly typical parkinsonism in 2000 (see ref. 11), similar families have been reported. Surprisingly, $\mathrm{Lu}$ et al. ${ }^{23}$ reported that four families with SCA2 were identified among 41 families with familial parkinsonism, about $10 \%$ of familial parkinsonism carried the expanded SCA2 CAG repeats in Taiwan people. Therefore, it is possible that the mutation rate of potential modifiers might account for the ethnic differences in the predisposition of parkinsonism-predominant SCA2. Better understanding of factors that determine a predominant parkinsonism phenotype in SCA2 may shed light on the pathogenesis of PD.

\section{ACKNOWLEDGEMENTS}

This work was supported by the Chinese National Natural Science Foundation (NO. 30400264) and the Yunnan Science and Technology Program (NO. 2008ZC068M). The authors thank the members of the Chinese family for their interest, support and cooperation in this study.

1 Jankovic, J. Parkinson's disease: clinical features and diagnosis. J. Neurol. Neurosurg. Psychiatry 79, 368-376 (2008).

2 de Rijk, M. C., Tzourio, C., Breteler, M. M., Dartigues, J. F., Amaducci, L., Lopez Pousa, S. et al. Prevalence of parkinsonism and Parkinson's disease in Europe: the EUROPARKINSON Collaborative Study. European community concerted action on the epidemiology of Parkinson's disease. J. Neurol. Neurosurg. Psychiatry 62, 10-15 (1997).

3 Polymeropoulos, M. H., Lavedan, C., Leroy, E., Ide, S. E., Dehejia, A., Dutra, A. et al. Mutation in the alpha-synuclein gene identified in families with Parkinson's disease. Science 276, 2045-2047 (1997).

4 Singleton, A. B., Farrer, M., Johnson, J., Singleton, A., Hague, S., Kachergus, J. et al. alpha-Synuclein locus triplication causes Parkinson's disease. Science 302, 841 (2003).
5 Di Fonzo, A., Tassorelli, C., De Mari, M., Chien, H. F., Ferreira, J., Rohe, C. F. et al. Comprehensive analysis of the LRRK2 gene in sixty families with Parkinson's disease. Europ. J. Hum. Genet. 14, 322-331 (2006).

6 Berg, D., Schweitzer, K., Leitner, P., Zimprich, A., Lichtner, P., Belcredi, P. et al. Type and frequency of mutations in the LRRK2 gene in familial and sporadic Parkinson's disease*. Brain 128, 3000-3011 (2005).

7 Kitada, T., Asakawa, S., Hattori, N., Matsumine, H., Yamamura, Y., Minoshima, S. et al. Mutations in the parkin gene cause autosomal recessive juvenile parkinsonism. Nature 392, 605-608 (1998).

8 Valente, E. M., Abou-Sleiman, P. M., Caputo, V., Muqit, M. M., Harvey, K., Gispert, S. et al. Hereditary early-onset Parkinson's disease caused by mutations in PINK1. Science 304, 1158-1160 (2004).

9 Bonifati, V., Rizzu, P., van Baren, M. J., Schaap, O., Breedveld, G. J., Krieger, E. et al. Mutations in the DJ-1 gene associated with autosomal recessive early-onset parkinsonism. Science 299, 256-259 (2003).

10 Sasaki, H., Wakisaka, A., Sanpei, K., Takano, H., Igarashi, S., Ikeuchi, T. et al. Phenotype variation correlates with CAG repeat length in SCA2-a study of 28 Japanese patients. J. Neurol. Sci. 159, 202-208 (1998).

11 Gwinn-Hardy, K., Chen, J. Y., Liu, H. C., Liu, T. Y., Boss, M., Seltzer, W. et al. Spinocerebellar ataxia type 2 with parkinsonism in ethnic Chinese. Neurology 55, 800-805 (2000).

12 Furtado, S., Payami, H., Lockhart, P. J., Hanson, M., Nutt, J. G., Singleton, A. A. et al. Profile of families with parkinsonism-predominant spinocerebellar ataxia type 2 (SCA2). Mov. Disord. 19, 622-629 (2004).

13 Shan, D. E., Soong, B. W., Sun, C. M., Lee, S. J., Liao, K. K. \& Liu, R. S. Spinocerebellar ataxia type 2 presenting as familial levodopa-responsive parkinsonism. Ann. Neurol. 50, 812-815 (2001).

14 Lu, C. S., Wu Chou, Y. H., Yen, T. C., Tsai, C. H., Chen, R. S. \& Chang, H. C. Doparesponsive parkinsonism phenotype of spinocerebellar ataxia type 2. Mov. Disord. 17, 1046-1051 (2002).

15 Furtado, S., Farrer, M., Tsuboi, Y., Klimek, M. L., de la Fuente-Fernandez, R., Hussey, J. et al. SCA-2 presenting as parkinsonism in an Alberta family: clinical, genetic, and PET findings. Neurology 59, 1625-1627 (2002).

16 Modoni, A., Contarino, M. F., Bentivoglio, A. R., Tabolacci, E., Santoro, M., Calcagni, M. L. et al. Prevalence of spinocerebellar ataxia type 2 mutation among Italian Parkinsonian patients. Mov. Disord. 22, 324-327 (2007).

17 Ruschendorf, F. \& Nurnberg, P. ALOHOMORA: a tool for linkage analysis using $10 \mathrm{~K}$ SNP array data. Bioinformatics 21, 2123-2125 (2005).

18 Abecasis, G. R., Cherny, S. S., Cookson, W. O. \& Cardon, L. R. GRR: graphical representation of relationship errors. Bioinformatics 17, 742-743 (2001).

19 O'Connell, J. R. \& Weeks, D. E. PedCheck: a program for identification of genotype incompatibilities in linkage analysis. Am. J. Hum. Genet. 63, 259-266 (1998).

20 Pulst, S. M., Nechiporuk, A., Nechiporuk, T., Gispert, S., Chen, X. N., Lopes-Cendes, I. et al. Moderate expansion of a normally biallelic trinucleotide repeat in spinocerebellar ataxia type 2. Nat. Genet. 14, 269-276 (1996).

21 Giunti, P., Sabbadini, G., Sweeney, M. G., Davis, M. B., Veneziano, L., Mantuano, E. et al. The role of the SCA2 trinucleotide repeat expansion in 89 autosomal dominant cerebellar ataxia families. Frequency, clinical and genetic correlates. Brain 121 (Part 3), 459-467 (1998).

22 Geschwind, D. H., Perlman, S., Figueroa, C. P., Treiman, L. J. \& Pulst, S. M. The prevalence and wide clinical spectrum of the spinocerebellar ataxia type 2 trinucleotide repeat in patients with autosomal dominant cerebellar ataxia. Am. J. Hum. Genet. 60, 842-850 (1997).

23 Lu, C. S., Wu Chou, Y. H., Kuo, P. C., Chang, H. C. \& Weng, Y. H. The parkinsonian phenotype of spinocerebellar ataxia type 2. Arch. Neurol. 61, 35-38 (2004).

24 Charles, P., Camuzat, A., Benammar, N., Sellal, F., Destee, A., Bonnet, A. M. et al. Are interrupted SCA2 CAG repeat expansions responsible for parkinsonism? Neurology 69, 1970-1975 (2007).

25 Sobczak, K. \& Krzyzosiak, W. J. CAG repeats containing CAA interruptions form branched hairpin structures in spinocerebellar ataxia type 2 transcripts. J. Biol. Chem. 280, 3898-3910 (2005).

26 Lin, J. X., Ishikawa, K., Sakamoto, M., Tsunemi, T., Ishiguro, T., Amino, T. et al. Direct and accurate measurement of CAG repeat configuration in the ataxin-1 (ATXN-1) gene by 'dual-fluorescence labeled PCR-restriction fragment length analysis'. J. Hum. Genet. 53, 287-295 (2008).

27 Cancel, G., Durr, A., Didierjean, O., Imbert, G., Burk, K., Lezin, A. et al. Molecular and clinical correlations in spinocerebellar ataxia 2: a study of 32 families. Hum. Mol. Genet. 6, 709-715 (1997).

28 Riess, O., Laccone, F. A., Gispert, S., Schols, L., Zuhlke, C., Vieira-Saecker, A. M. et al. SCA2 trinucleotide expansion in German SCA patients. Neurogenetics. 1, 59-64 (1997).

29 Almaguer-Mederos, L. E., Falcon, N. S., Almira, Y. R., Zaldivar, Y. G., Almarales, D. C. Gongora, E. M. et al. Estimation of the age at onset in spinocerebellar ataxia type 2 Cuban patients by survival analysis. Clin. Genet. 78, 169-174.

30 Gispert, S., Twells, R., Orozco, G., Brice, A., Weber, J., Heredero, L. et al. Chromosomal assignment of the second locus for autosomal dominant cerebellar ataxia (SCA2) to chromosome 12q23-24.1. Nat. Genet. 4, 295-299 (1993). 